\title{
Constant envelope signal space diversity
}

T.V.K. Chaitanya, Danyo Danev and Erik G. Larsson

\section{Linköping University Post Print}

\section{Tweet}

N.B.: When citing this work, cite the original article.

(C2014 IEEE. Personal use of this material is permitted. However, permission to reprint/republish this material for advertising or promotional purposes or for creating new collective works for resale or redistribution to servers or lists, or to reuse any copyrighted component of this work in other works must be obtained from the IEEE.

T.V.K. Chaitanya, Danyo Danev and Erik G. Larsson, Constant envelope signal space diversity, 2014, Proceedings of the 2014 IEEE International Conference on Acoustics, Speech and Signal Processing (ICASSP), 3147-3151.

http://dx.doi.org/10.1109/ICASSP.2014.6854180

Postprint available at: Linköping University Electronic Press

http://urn.kb.se/resolve?urn=urn:nbn:se:liu:diva-109639 


\title{
CONSTANT ENVELOPE SIGNAL SPACE DIVERSITY
}

\author{
Tumula V. K. Chaitanya*1, Danyo Danev ${ }^{2}$, and Erik G. Larsson ${ }^{2}$ \\ ${ }^{1}$ Dept. of Electrical and Computer Engineering, McGill University, Montreal, Canada. \\ ${ }^{2}$ Dept. of Electrical Engineering (ISY), Linköping University, Linköping, Sweden.
}

\begin{abstract}
We propose a nonlinear signal space diversity (SSD) precoding technique that produces transmit signals that have constant envelope (CE) in discrete time, resulting in low peak-to-average power ratio (PAPR) waveforms after pulse-shape filtering. We propose two methods for construction of CE signal set. While the proposed CESSD scheme is inferior to the conventional SSD designs in terms of coding gain performance, it performs better in terms of overall power efficiency because of the reduced back-off requirement of the power amplifier (PA).
\end{abstract}

Index Terms - Signal space diversity, constant envelope, constellation design, power amplifier efficiency, low PAPR.

\section{INTRODUCTION}

One important reason for the low power efficiency of the current wireless communication systems is the use of signals with high PAPR values. To support the transmission of signals with high PAPR values, highly linear PAs have to be used with a back-off value proportional to the PAPR of the input signals. Operating with a back-off reduces the amplifier efficiency, hence constant envelope (CE) signals are the most desirable from the power efficiency point of view.

Signal space diversity (SSD) is a technique originally proposed in $[1,2]$ for extracting the diversity available in the channel and thereby improving the reliability of transmission over fading channels. SSD has found applications in various branches of wireless communications, for example, to extract multipath diversity in orthogonal frequency division multiplexing (OFDM) systems [3], and in space-time coded systems to extract antenna diversity $[4,5]$. In practical systems like LTE, SSD can improve the performance of the uplink control signaling $[6,7]$. SSD is most helpful when the outer channel code is short or weak [8], which is the case in practice for example when transmitting short data blocks on the control plane.

One of the drawbacks of the conventional SSD designs [1]-[8] is that the precoded symbols do not generally have constant envelope even if the input symbols do. Even though the SSD technique results in an improved performance, previous works did not consider the effect of the reduction in the PA efficiency when using the SSD precoding. In this work, we propose a nonlinear SSD precoding technique that results in transmit signals that have a CE. Because of the $\mathrm{CE}$ property, the proposed design reduces the back-off required in the PA, thereby improving the PA efficiency. Our work differs from the work in [9], in which CE precoding design was proposed for large antenna systems with the assumption of perfect channel state information (CSI) knowledge at the transmitter. Whereas our

* The first author performed the work when he was with the Dept. of Electrical Engineering (ISY), Linköping University, Linköping, Sweden.

This work was supported in part by Ericsson, VINNOVA, the Swedish Foundation for Strategic Research (SSF), and ELLIIT. precoding design does not require CSI knowledge at the transmitter.

\section{SYSTEM MODEL AND THE CONVENTIONAL SSD}

We consider a system model similar to the one used in [2]. Let $\mathbf{s}=\left(s_{1}, \cdots, s_{n}\right)^{T}$ denote an $n$-dimensional column vector with $s_{i} \in \mathcal{A}$, for $1 \leq i \leq n$, where $\mathcal{A} \subset \mathbb{C}$ denotes a normalized two-dimensional constellation like M-QAM or M-PSK with unit average energy. Assuming that $|\mathcal{A}|=M$, we have $\mathbf{s} \in \mathcal{A}^{n} \triangleq$ $\left\{\mathbf{s}_{1}, \cdots, \mathbf{s}_{N}\right\} \subset \mathbb{C}^{n}$ with $N=M^{n}$. In the case of a system using conventional (linear) SSD techniques [1]-[7], the symbol vector $\mathbf{s}$ is multiplied by an $n \times n$ precoding matrix $\boldsymbol{\Psi}$, such that we obtain the transmitted precoded data vector $\mathbf{x} \in \mathcal{X} \triangleq\left\{\mathbf{x}_{1}, \cdots, \mathbf{x}_{N}\right\}$ as $\mathbf{x}=\Psi \mathbf{s}$. We can write the received signal vector as $\mathbf{y}=$ $\sqrt{\left(P_{\text {out }} / n\right)} \mathbf{h} \odot \mathbf{x}+\mathbf{z}$ where $P_{\text {out }}$ denotes the output power of the PA, and $\mathbf{h}=\left(h_{1}, \cdots, h_{n}\right)^{T}$ and $\mathbf{z}=\left(z_{1}, \cdots, z_{n}\right)^{T}$ are the channel gain vector and the noise vector, respectively. The notation $\odot$ represents the element-wise product. We assume that $h_{i}$ and $z_{i}, 1 \leq i \leq n$ are i.i.d. with $h_{i} \sim \mathcal{C N}(0,1)$ and $z_{i} \sim \mathcal{C N}\left(0, N_{0}\right)$. Assuming perfect CSI at the receiver, the pairwise error probability (PEP), which is the probability that the transmitted vector $\mathbf{s}_{a} \in \mathcal{A}^{n}$ is erroneously decoded as $\mathbf{s}_{b} \neq \mathbf{s}_{a}$ can be upper bounded as [2, 3]:

$$
\begin{aligned}
\operatorname{PEP}\left(\mathbf{s}_{a} \rightarrow \mathbf{s}_{b}\right) & \leq \prod_{i=1}^{n}\left(1+\frac{P_{\text {out }}\left|x_{a, i}-x_{b, i}\right|^{2}}{4 n N_{0}}\right)^{-1} \\
& \leq\left(\frac{P_{\text {out }}}{4 n N_{0}}\left(\prod_{\substack{i=1 \\
x_{a, i} \neq x_{b, i}}}^{r_{e}}\left|x_{a, i}-x_{b, i}\right|^{2}\right)^{\frac{1}{r_{e}}}\right)^{-r_{e}},
\end{aligned}
$$

where $r_{e} \triangleq \operatorname{Rank}\left(\mathbf{X}_{a b}\right), \quad \mathbf{X}_{a b} \triangleq$ $\operatorname{diag}\left(\left|x_{a, 1}-x_{b, 1}\right|^{2}, \cdots,\left|x_{a, n}-x_{b, n}\right|^{2}\right) \quad$ with $\quad \mathbf{x}_{a} \triangleq$ $\left(x_{a, 1}, \cdots, x_{a, n}\right)^{T}=\Psi_{\mathbf{s}_{a}}$ and $\mathbf{x}_{b} \triangleq\left(x_{b, 1}, \cdots, x_{b, n}\right)^{T}=\Psi_{\mathbf{s}_{b}}$. As in [3], we call $r_{e}$ the pairwise diversity order $G_{d, e}$ and the term $\left(\prod_{\substack{i=1 \\ x_{a, i} \neq x_{b, i}}}^{r_{e}}\left|x_{a, i}-x_{b, i}\right|^{2}\right)^{\frac{1}{r_{e}}}$ the pairwise coding gain $G_{c, e}$. Note that $G_{d, e}$ and $G_{c, e}$ depend on the error vector $\mathbf{e}=\mathbf{s}_{a}-\mathbf{s}_{b}$ through $\mathbf{u}_{e} \triangleq \mathbf{x}_{a}-\mathbf{x}_{b}=\mathbf{\Psi}$. Let the set of all possible error vectors be denoted as $\mathcal{E} \triangleq\left\{\mathbf{s}_{a}-\mathbf{s}_{b} \mid \mathbf{s}_{a}, \mathbf{s}_{b} \in \mathcal{S}, \mathbf{s}_{b} \neq \mathbf{s}_{a}\right\}$. The diversity and coding gains of an SSD technique are defined as:

$$
G_{d}(\mathcal{X}) \triangleq \min _{\mathbf{e} \in \mathcal{E}} G_{d, e}=\min _{\mathbf{e} \in \mathcal{E}} r_{e} \text { and } G_{c}(\mathcal{X}) \triangleq \min _{\mathbf{e} \in \mathcal{E}} G_{c, e} .
$$

In order to minimize the PEP, we should have $G_{d}(\mathcal{X})=n$ and maximize $G_{c}(\mathcal{X})$. In addition, it is usually desirable that $\Psi$ is unitary so that performance in the absence of fading is unaffected by the precoding. When $G_{d}(\mathcal{X})=n$, the coding gain is related to the minimum product distance defined by $\operatorname{dp}_{\min }(\mathcal{X}) \triangleq$ 
Table 1. $\rho\left(\mathcal{X}_{\mathcal{P}}\right), \rho_{c}$ and $G_{c}(\mathcal{X})$ in $\mathrm{dB}$ for different SSD schemes with $\mathcal{A}$ being a QPSK constellation

\begin{tabular}{|c|c|c|c|c|c|}
\hline \multirow{2}{*}{} & \multirow{2}{*}{$\rho_{d}\left(\mathcal{X}_{\mathcal{P}}\right)$} & \multicolumn{3}{|c|}{$\rho_{c}$} & \multirow{2}{*}{$G_{c}(\mathcal{X})$} \\
\cline { 3 - 5 } & & $\alpha=0.1$ & $\alpha=0.3$ & $\alpha=0.5$ & \\
\hline \hline$n=2$ & 2.3 & 5.2 & 4.7 & 4.0 & 0.0 \\
\hline$n=3$ & 4.4 & 7.1 & 6.6 & 6.0 & -2.1 \\
\hline$n=4$ & 5.2 & 7.9 & 7.4 & 6.7 & -3.0 \\
\hline
\end{tabular}

$\min _{\mathbf{x}_{a}, \mathbf{x}_{b} \in \mathcal{X}} \prod_{i=1}^{n}\left|x_{a, i}-x_{b, i}\right|$ as $G_{c}(\mathcal{X})=\left(\operatorname{dp}_{\min }(\mathcal{X})\right)^{\frac{2}{n}}$. In [2], orthonormal $\Psi$ matrices were specified, that achieve $G_{d}(\mathcal{X})=$ $n$ and maximize $G_{c}(\mathcal{X})$. However, in [3, 4], it was shown that by using unitary $\boldsymbol{\Psi}$ matrices, one can retain the property $G_{d}(\mathcal{X})=n$ with an increase in the value of achievable $G_{c}(\mathcal{X})$ relative to the case when the matrix $\boldsymbol{\Psi}$ only contains real-valued entries. In [3, 4], unitary designs with $\Psi \Psi^{H}=\mathbf{I}_{n}$ were specified for even values of $n$. For odd values of $n$, non-unitary designs were specified with the property $\operatorname{tr}\left(\Psi \Psi^{H}\right)=n$.

\section{PAPR OF THE CONVENTIONAL SSD DESIGNS}

The SSD designs in [1]-[6] have a drawback in the sense that the precoded output data vector $\mathbf{x}$ does not contain constant modulus symbols even if the input symbols in $\mathbf{s}$ have constant modulus. Let $\mathcal{X}_{\mathcal{P}} \triangleq\left\{x_{1}, x_{2}, \ldots, x_{K}\right\} \subset \mathbb{C}$ denote the set of distinct precoded output data symbols. We define the discrete-time PAPR of the set $\mathcal{X}_{\mathcal{P}}$ as $\rho_{d}\left(\mathcal{X}_{\mathcal{P}}\right) \triangleq\left(\max _{i}\left|x_{i}\right|^{2}\right) /\left(\frac{1}{K} \sum_{i}\left|x_{i}\right|^{2}\right)$ and the PAPR of the continuous-time signal after pulse-shape filtering as $\rho_{c} \triangleq \max \left(|x(t)|^{2}\right) / \mathbb{E}\left(|x(t)|^{2}\right)$. Table 1 shows a comparison of $\rho_{\mathrm{d}}\left(\mathcal{X}_{\mathcal{P}}\right), \rho_{c}$ and $G_{c}(\mathcal{X})$ in $\mathrm{dB}$ for the conventional SSD schemes with complex-valued $\Psi$ matrices for different values of $n$ and with $\mathcal{A}$ corresponding to a QPSK constellation. ${ }^{1}$ For computing $\rho_{c}$, we used root-raised cosine pulse-shaping filter with different values of roll-off factor $\alpha$, a delay of 10 symbol durations and an oversampling factor of 16 . We computed the complementary cumulative distribution function (CCDF) of instantaneous PAPR value over a block of 50 modulation symbols generated randomly, and considered the $10^{-4}$ point in the CCDF curve as the PAPR value of a given precoding scheme. Since the SSD designs in the literature result in a PAPR value higher than $0 \mathrm{~dB}$, the PA needs to be backed off to ensure we operate in its linear regime. The power amplifier efficiency as a function of the PAPR of the transmitted signals can be written as [10]:

$$
\eta \triangleq \frac{P_{\text {out }}}{P_{\text {in }}}=\frac{\xi}{\rho_{c}}
$$

where $P_{\text {out }}$ and $P_{\text {in }}$ denote the output and input power values of the PA, respectively, $\xi$ corresponds to the drain efficiency of the PA and $\rho_{c}$ denotes the PAPR value of the PA input signal. The model in (2) is valid for class A PAs and reflects the fact that as the value of $\rho_{c}$ increases, the operating point of the PA should be backed off by $10 \log _{10}\left(\rho_{c}\right) \mathrm{dB}$ to avoid clipping of the signal. As we can see from (2), the PA efficiency reduces inversely proportional to the increase in the PAPR value. ${ }^{2}$ The maximum value of $\xi$ for class A PAs is 0.5 .

\footnotetext{
${ }^{1}$ The $\boldsymbol{\Psi}$ matrices for $n=2$ and 4 are taken from [3]. These are the optimal linear precoding matrices in terms of maximizing the minimum product distance. For the case of $n=3$, we used the precoding matrix specified in [4], which was found by a computer search.

${ }^{2}$ For class B amplifiers, the efficiency can be written as $\eta=\xi / \sqrt{\rho_{c}}$. However, in this study we consider class A amplifiers, because: i) they are preferred as broadband power amplifiers at $\mathrm{GHz}$ frequencies; ii) power gain is higher compared to class B amplifiers [10].
}

\section{PROPOSED CONSTANT ENVELOPE SSD}

Motivated by the discussion about PA efficiency and PAPR of the input signal in Section 3, we propose a new nonlinear SSD technique which gives precoded output symbols with constant modulus. The design goals are: i) to achieve the maximum possible diversity gain $n$; ii) to maximize the minimum product distance; and iii) to yield precoded output symbols with constant modulus. Note that our focus here is on systems that use no or relatively weak channel coding. Then, minimizing the average error probability is relevant. By contrast, for systems that use strong channel coding, maximizing other metrics like the mutual information [11], may be more appropriate. Hence, in this work, we are interested in the construction of a set $\mathcal{T}=\left\{\mathbf{t}_{1}, \mathbf{t}_{2}, \cdots, \mathbf{t}_{N}\right\} \subset \mathbb{C}^{n}$, which corresponds to the set of precoder output vectors. The first requirement of achieving diversity gain $n$ corresponds to the following condition:

$$
\operatorname{dp}_{\min }(\mathcal{T}) \triangleq \min _{\substack{\mathbf{t}_{a}, \mathbf{t}_{b} \in \mathcal{T} \\ \mathbf{t}_{a} \neq \mathbf{t}_{b}}} \prod_{i=1}^{n}\left|t_{a, i}-t_{b, i}\right| \neq 0 .
$$

In (3), $d p_{\min }(\mathcal{T})$ denotes the minimum product distance of the set $\mathcal{T}$. The second requirement is to maximize $\operatorname{dp}_{\min }(\mathcal{T})$. The third requirement corresponds to choosing all elements of $\mathbf{t}_{m} \in \mathcal{T}, 1 \leq$ $m \leq N$ from the unit circle. In the proposed precoding method, we transmit the components of the corresponding precoded vector $\mathbf{t}_{m} \in \mathcal{T}$ on the channel. One can view the proposed CE-SSD as a direct mapping from the input bits to the precoded vectors $\mathbf{t}_{m}$. Now we propose two specific constructions of a set $\mathcal{T}$ that meets the three requirements discussed above.

\subsection{Construction - I}

In this construction, in order to satisfy the unit modulus constraint for the elements of $\mathbf{t}_{m} \in \mathcal{T}$, we choose $\mathbf{t}_{m}=$ $\left(e^{j \phi_{n m-n+1}}, \cdots, e^{j \phi_{n m}}\right)^{T}$, for $1 \leq m \leq N$, with $0 \leq \phi_{i} \leq$ $2 \pi, 1 \leq i \leq N n$. To maximize the coding gain advantage $G_{c}(\mathcal{T})$, we need to find the $\mathcal{T}$ that maximizes $\operatorname{dp}_{\min }(\mathcal{T})$. We formulate the corresponding optimization problem as:

$$
\begin{aligned}
& \max \gamma \\
& \text { sub. to } \prod_{i=1}^{n}\left|e^{j \phi_{n a-n+i}}-e^{j \phi_{n b-n+i}}\right| \geq \gamma, \forall a \neq b \in\{1, \cdots, N\} \\
& \quad 0 \leq \phi_{i} \leq 2 \pi, 1 \leq i \leq N n
\end{aligned}
$$

The first constraint in (4) corresponds to a total number of $\left(\begin{array}{c}N \\ 2\end{array}\right)$ individual constraints. The total number of variables $\phi_{(.)}$in the optimization problem is $n N=n M^{n}$, which grows exponentially with the dimension of the transmitted signal vector $n$. Since (4) is a nonlinear and non-convex problem, we are not guaranteed to find the globally optimal solution unless an exhaustive search is performed over the optimization variables. However the complexity of such an exhaustive search is prohibitively high. For $n=2$ and $M=4$, a solution to the optimization problem in (4) can be obtained using standard nonlinear optimization algorithms. For $n \geq 3$ and $M \geq 4$, the computational complexity of finding even a local optimum using this construction is very high as the number of optimization variables is at least 192. Moreover, the transmitter needs to store $n M^{n}$ real values corresponding to the angles in the memory to implement the proposed CE-SSD technique.

\subsection{Construction - II}

In this construction, we limit ourselves to choose the elements of $\mathbf{t}_{m}, 1 \leq m \leq N$, from $N$ points uniformly spaced on the unit circle 
and with phase angles given by $\phi_{m}=(m-1) \pi / N,, 1 \leq m \leq N$. If we view all the $\mathbf{t}_{m}$ vectors of $\mathcal{T}$ together as columns of a matrix $\mathbf{T}$ of size $n \times N$, the problem of constructing the set $\mathcal{T}$ now reduces to assigning each element of the $\mathbf{T}$ matrix to an integer $m, 1 \leq m \leq N$ corresponding to the angle. The condition of achieving maximum diversity order in (3) translates to having different integers corresponding to the angles in each row of the $\mathbf{T}$ matrix. In other words, all the integers in each row of the $\mathbf{T}$ matrix should form a permutation of the vector $(1, \cdots, N)$. Let $\Pi_{i}$ denote the permutation corresponding to the $i$ th row of $\mathbf{T}$. Without loss of generality, we can assume that $\Pi_{1}=(1, \cdots, N)$, and the optimization problem of maximizing $\operatorname{dp}_{\min }(\mathcal{T})$ can now be written as:

$$
\max _{\Pi_{2}, \cdots, \Pi_{n}} \min _{a, b \in\{1, \cdots, N\}} \prod_{\substack{a \neq b \\ a \neq 1}}^{n}\left|e^{j \phi_{\Pi_{i}(a)}}-e^{j \phi_{\Pi_{i}}(b)}\right|,
$$

where $\Pi_{i}(a) \in\{1, \cdots, N\}$ denotes the $a$ th element of permutation $\Pi_{i}$. An exhaustive search for the solution to (5) involves searching over $\approx((N-1) !)^{n-1}$ combinations, which is a very large number even for $n=2$ and $M=4$. In order to reduce the size of the search space for larger values of $n$, we limit ourselves to the space of the permutations that belong to a specific class, i.e.,

$$
\begin{aligned}
\Pi_{2}, \ldots, \Pi_{n} \in\left\{\Pi^{k} \mid \Pi^{k}(m)\right. & =(1+(m-1) k) \bmod N \\
\text { and } \operatorname{gcd}(k, N) & =1\} .
\end{aligned}
$$

The condition of $\operatorname{gcd}(k, N)=1$, i.e., $k$ and $N$ being relatively prime, guarantees that $\Pi^{k}$ is a permutation of the vector $(1, \cdots, N)$. The total number of permutations in the reduced search space is given by $(\varphi(N))^{n-1}$, where $\varphi(N)$ is Euler's totient function. For example, with $M=4$ and $n=2,3$, and 4 , we have $N=$ 16,64 , and 256 , respectively. For these values of $N, \varphi(N) \ll$ $(N-1)$ !. The motivation for selecting the permutations as in (6) is that: i) the storage requirement is minimal as the transmitter needs to store only the values of $k$ corresponding to $\Pi_{2}, \cdots, \Pi_{n}$ and the value of $N$; ii) the number of permutations in the search space is much less than that for the exhaustive search; and iii) as we see in Section 5 , the solution obtained from the reduced search space is close to the optimal solution for the case of $n=2$ found using a simplified search space algorithm described below.

The idea of the simplified search algorithm for the case of $n=2$ is to construct a permutation $\Pi_{2}$ such that we avoid all minimum product distance values smaller than a given value, lets say $d$. The pseudo-code of the simplified algorithm is given below.

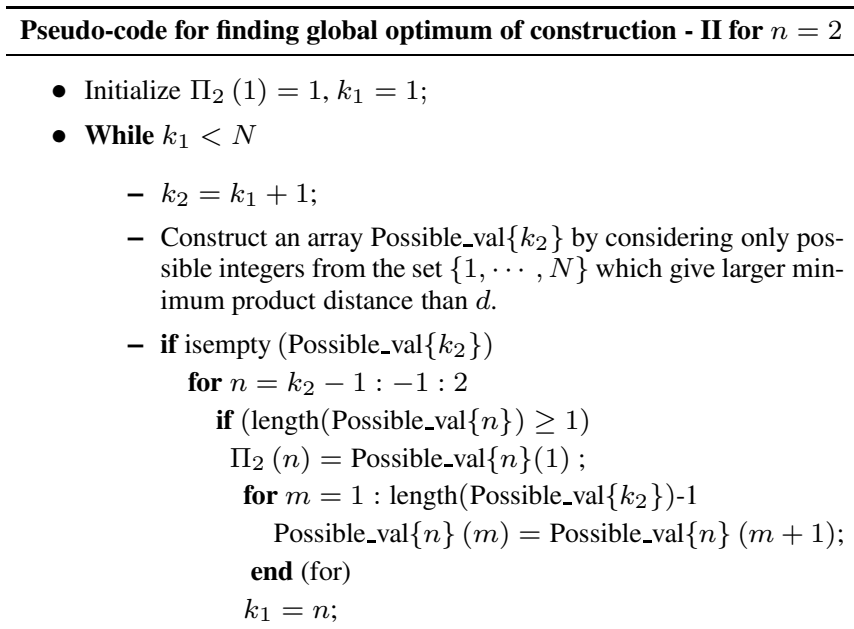

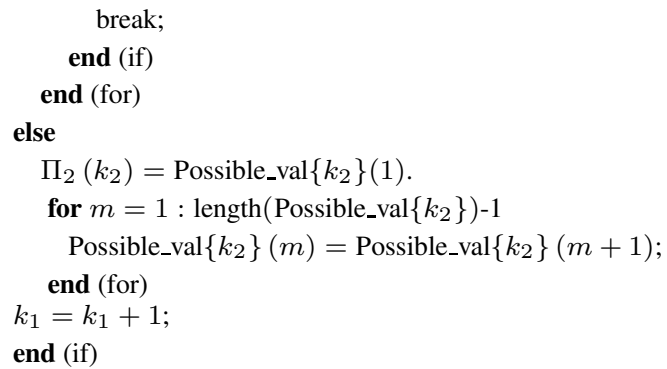

end (While)

Note that for $n \geq 3$, the complexity of using a similar simplified algorithm to find optimal permutations is also prohibitively high.

\subsection{Discussion}

From the receiver point of view, the proposed CE-SSD technique has the same complexity as the conventional SSD technique. To perform optimal detection, the receiver has to search through the $M^{n}$ possible transmitted signal vectors. For the conventional SSD, suboptimal detection methods such as ZF, MMSE as well as the sphere decoding method [12] can be used. However, these suboptimal detection methods cannot be applied to the proposed CE-SSD method. On the transmitter side, in case of conventional SSD, the transmitter can either store the rotated constellations in memory or only the complex-valued $\Psi$ matrix and generate the precoded symbol vector $\mathbf{x}$ by performing multiplications. For the proposed CE-SSD precoding using construction-II, the transmitter needs to store only $n$ integer values in the memory. In case of the non-SSD, we can detect the elements of s separately and hence it is less complex and there is no additional storage requirement at the transmitter. In case of a coded transmission with the proposed CE-SSD scheme, one has to consider optimal mapping of input bits to the precoded vectors $\mathbf{t}_{m}, 1 \leq m \leq N$. However, such a study is outside the scope of present work. For presenting the results for a coded system in Section 5, we used a Gray mapping of input bits to the precoded vectors for the CE-SSD scheme.

Even though the discrete-time PAPR $\rho_{d}(\mathcal{T})$ of the proposed constructions is $0 \mathrm{~dB}$, the values of $\rho_{c}$ for the proposed CE-SSD scheme are 3.1, 2.5, and $1.8 \mathrm{~dB}$ after pulse-shape filtering with $\alpha=0.1,0.3$, and 0.5 , respectively. The values of $\rho_{c}$ for the proposed CE-SSD are independent of the value of $n$, and are better than the values for the conventional method, cf. Table 1 . In general, we can construct the $N$ vectors of the set $\mathcal{T}$ by considering any $p, N \leq p \leq n N$ elements on the unit circle. In a way, constructions I and II considered in this work represent the two extreme cases of possible mappings.

\section{RESULTS}

In this section we present results comparing the performance of our proposed CE-SSD technique with conventional SSD and non-SSD schemes. For the conventional SSD, we use the complex-valued $\Psi$ matrices for comparison. The results are presented for the case of $\mathcal{A}$ being a standard QPSK constellation and for different values of $n$.

Coding Gain Comparison: For $n=2$, we present the result for both constructions-I and II in terms of the coding gain. For construction-I, we found the vectors in the set $\mathcal{T}$ by solving (4) with many random initializations and using the the sequential quadratic programming (SQP) method. The angles in radians for the elements of set $\mathcal{T}$ that we obtained when maximizing $\operatorname{dp}_{\min }(\mathcal{T})$ are shown in Table 2. For this set $\mathcal{T}$, $\operatorname{dp}_{\min }^{\operatorname{method}-\mathrm{I}}(\mathcal{T})$ is obtained as 0.64 . For 
Table 2. Angles in radians for set $\mathcal{T}$ obtained using construction - I.

\begin{tabular}{|c|c|c|c|c|c|c|c|}
\hline$i$ & $\phi_{i}$ & $i$ & $\phi_{i}$ & $i$ & $\phi_{i}$ & $i$ & $\phi_{i}$ \\
\hline \hline 1 & 3.09 & 9 & 3.82 & 17 & 2.31 & 25 & 3.42 \\
\hline 2 & 2.76 & 10 & 4.39 & 18 & 5.10 & 26 & 0.00 \\
\hline 3 & 5.80 & 11 & 6.21 & 19 & 0.27 & 27 & 4.57 \\
\hline 4 & 4.77 & 12 & 1.34 & 20 & 3.87 & 28 & 3.48 \\
\hline 5 & 1.56 & 13 & 1.23 & 21 & 2.67 & 29 & 4.94 \\
\hline 6 & 0.40 & 14 & 3.16 & 22 & 1.01 & 30 & 5.56 \\
\hline 7 & 1.99 & 15 & 5.46 & 23 & 4.15 & 31 & 0.64 \\
\hline 8 & 2.08 & 16 & 2.41 & 24 & 1.72 & 32 & 5.94 \\
\hline
\end{tabular}

Table 3. Performance comparison in $\mathrm{dB}$ between the proposed CESSD method and the conventional SSD method.

\begin{tabular}{|c|c|c|c|}
\hline \multicolumn{2}{|c|}{} & $\begin{array}{c}\text { Conventional } \\
\text { SSD }\end{array}$ & CE-SSD \\
\hline \hline \multirow{3}{*}{$n=2$} & Coding gain $\left(G_{c}\right)$ & 0 & -1.8 \\
\cline { 2 - 4 } & PA eff. $(\eta)$ & -7.7 & -5.5 \\
\cline { 2 - 4 } & Overall eff. $\left(G_{c} \eta\right)$ & $-\mathbf{7 . 7}$ & $-\mathbf{7 . 3}$ \\
\hline \multirow{3}{*}{$n=3$} & Coding gain $\left(G_{c}\right)$ & -2.1 & -5.1 \\
\cline { 2 - 4 } & PA eff. $(\eta)$ & -9.6 & -5.5 \\
\cline { 2 - 4 } & Overall eff. $\left(G_{c} \eta\right)$ & $\mathbf{- 1 1 . 7}$ & $\mathbf{- 1 0 . 6}$ \\
\hline \multirow{3}{*}{$n=4$} & Coding gain $\left(G_{c}\right)$ & -3.0 & -7.1 \\
\cline { 2 - 4 } & PA eff. $(\eta)$ & -10.4 & -5.5 \\
\cline { 2 - 4 } & Overall eff. $\left(G_{c} \eta\right)$ & $\mathbf{- 1 3 . 4}$ & $\mathbf{- 1 2 . 6}$ \\
\hline
\end{tabular}

construction-II, using the reduced search space, we find the optimal $k$ value for $\Pi_{2}$ in (6) as 9. This gave us a minimum product distance value of 0.59 . Using the simplified global optimum search algorithm outlined in Section 4.2, we found the optimal $\Pi_{2}$ of the whole feasible set, and is given by

$$
\Pi_{2}^{\mathrm{opt}}=[1,6,12,3,8,15,5,10,2,13,7,16,11,4,14,9] .
$$

For construction-II, with $\Pi_{2}^{\text {opt }}$, we obtain the value of $\mathrm{dp}_{\min }^{\text {method }-\mathrm{II}}(\mathcal{T})$ as 0.65

For the cases of $n=3$ and 4 , we limit ourselves to constructionII with the reduced search space constraint specified in (6). Finding the solution using construction-I is complex as the number of optimization variables for $n=3$ and 4 are 192 and 1024, respectively. In the case of $n=3$, we found the optimal values of $k$ for $\Pi_{2}$ and $\Pi_{3}$ as 15 and 29, respectively. The minimum product distance for the $n=3$ case with the proposed construction is obtained as 0.17 . For the case of $n=4$, we found the optimal values of $k$ for $\Pi_{2}, \Pi_{3}$ and $\Pi_{4}$ as 29,97 and 107 , respectively. The minimum product distance value obtained in this case is 0.038 . Note that even though we do not know the globally optimum minimum product distance values that can be obtained with the proposed CE-SSD construction for $n \geq 3$, the proposed sub-optimal solutions can be viewed as lower bounds and they provide gains over the conventional SSD in terms of the overall power efficiency as shown in Table 3.

Overall Efficiency Comparison: Table 3 shows an analytical performance comparison between the proposed CE-SSD technique and the conventional SSD designs with a complex-valued $\boldsymbol{\Psi}$ matrix in terms of the coding gain and the PA efficiency (using pulseshaping filter with roll-off factor $\alpha=0.3$ ). As seen from Table 3, in terms of the coding gain, the proposed method is inferior to the linear SSD designs. This is expected as we limit ourselves to searching for constellation points on the unit circle in our proposed method. However, the proposed method gains in terms of the PA efficiency because of the reduced value of $\rho_{c}$. If we consider the overall power

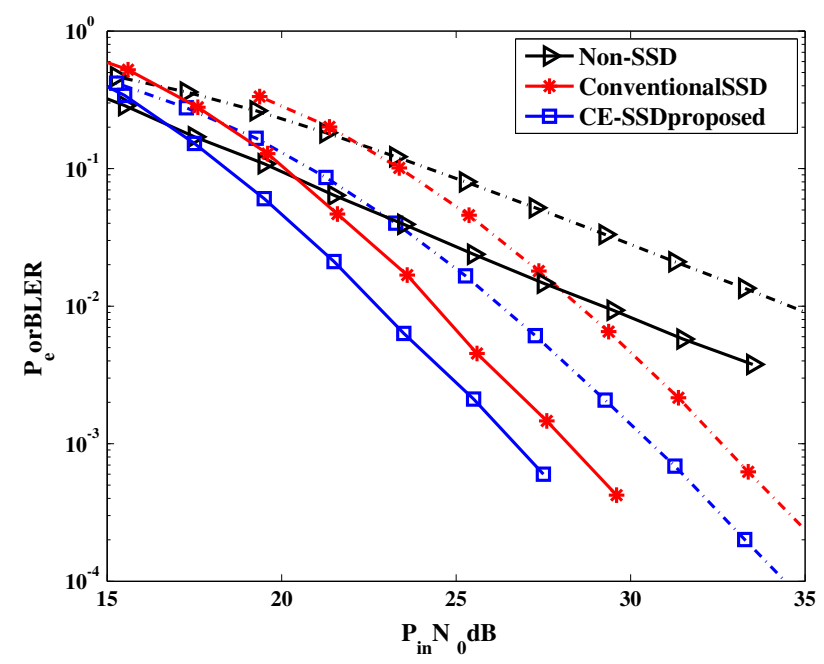

Fig. 1. Performance comparison for different methods, $n=3$. Solid lines correspond to a comparison in a coded system and dashed lines in an uncoded system. For the coded system, we considered a rate 1/2-convolutional code with $G=(2335)$ and constraint length 5 . Each codeword experiences three independent channels. We pick one modulation symbol from each of the codeword parts experiencing independent fading and apply the SSD scheme.

efficiency by taking into account the coding gain performance together with the required PA back-off, see (2), then with a linear PA, the CE-SSD technique can provide gains of $0.4,1.1$ and $0.8 \mathrm{~dB}$ for $n=2,3$ and 4 , respectively.

Error Rate Performance Comparison: The results presented in Table 3 are based on the upper bound of the PEP. Fig. 1 shows an error rate performance comparison of different schemes for the case of $n=3$. We compared vector error rate $\left(P_{e}\right)$ in an uncoded system and block-error rate (BLER) in a coded system. For the uncoded case, the analytically predicted gains are also seen in the actual error rate performance. Even in case of a coded system, conventional SSD schemes were shown to provide gains over non-SSD transmissions if the channel code is not strong enough to fully extract the diversity available in the channel resources $[6,7,8]$. Weak channel codes are used in practical systems because of constraints induced by the application. ${ }^{3}$ For the performance comparison in a coded system, we considered a weak rate-1/2 convolutional code with $G=\left(\begin{array}{ll}23 & 35\end{array}\right)$ and constraint length 5 , and the CE-SSD can provide gains in this case as well.

\section{CONCLUSIONS}

The new CE-SSD technique proposed in this work brings up to 1.1 $\mathrm{dB}$ power gains in terms of overall power efficiency compared to the conventional SSD. The proposed designs are however strictly sub-optimal because of the search over reduced feasible sets. For construction-II, one might find better designs by optimizing the constellation points together with the permutations search. A possible extension of the proposed work is to consider new SSD mappings with a relaxation of constant envelope constraint and the resulting trade-off between coding gain and PAPR of the transmitted signal.

\footnotetext{
${ }^{3}$ For instance, certain modes of control signaling information transmission in LTE systems use a Reed-Muller code.
} 


\section{REFERENCES}

[1] V. M. DaSilva and E. S. Sousa, "Fading-resistant modulation using several transmitter antennas," IEEE Trans. Commun., vol. 45, pp. 1236-1244, Oct. 1997.

[2] J. Boutros and E. Viterbo, "Signal space diversity, a power- and bandwidth-efficient diversity technique for the Rayleigh fading channel," IEEE Trans. Inform. Theory, vol. 44, pp. 1453-1467, July 1998.

[3] Z. Liu, Y. Xin, and G. B. Giannakis, "Linear constellation precoding for OFDM with maximum multipath diversity and coding gains," IEEE Trans. Commun., vol. 51, pp. 416-427, Mar. 2003.

[4] Y. Xin, Z. Wang, and G. B. Giannakis, "Space-time diversity systems based on linear constellation precoding," IEEE Trans. Wireless Commun., vol. 2, no. 2, pp. 294-309, Mar. 2003.

[5] H. El Gamal and M. O. Damen, "Universal space-time coding," IEEE Trans. Inf. Theory, vol. 49, no. 5, pp. 1097-1119, May 2003.

[6] T. V. K. Chaitanya and E. G. Larsson, "Improving 3GPP-LTE uplink control signaling performance using complex-field coding," IEEE Trans. Vech. Tech., vol. 62, no. 1, pp. 161-171, Jan. 2013.
[7] T. V. K. Chaitanya, E. G. Larsson, and N. Wiberg, "Improved error protection for uplink control signaling in 3GPP-LTE via complex-field coding," Proc. of the IEEE Vehicular Technology Conference (VTC), May 2010.

[8] R. Wang, X. Ma, and G. B. Giannakis, "Improving the performance of coded FDFR multi-antenna systems with turbodecoding," Wireless Communications Mob. Computing, vol. 4, no. 7, pp. 711-725, Nov. 2004.

[9] S. K. Mohammed and E. G. Larsson, "Single-user beamforming in large-scale MISO systems with per-antenna constantenvelope constraints: the Doughnut channel," IEEE Trans. Wireless Commun., vol. 11, no. 11, pp. 3992-4005, Nov. 2012.

[10] J. L. B. Walker, Handbook of RF and Microwave Power Amplifiers. Cambridge Univ. Press, 2011.

[11] Q. Xie et al., "Coded modulation with signal space diversity," IEEE Trans. Wireless Commun., vol. 10, no. 2, pp. 660-669, Feb. 2011.

[12] E. Viterbo and J. Boutros, "A universal lattice code decoder for fading channels," IEEE Trans. Inform. Theory, vol. 45, pp. 1639-1642, July 1999. 